Mini-Review

\title{
Infiltrating Lobular Carcinoma of the Breast Presenting as Gastrointestinal Obstruction: A Mini Review
}

\author{
P Carcoforo', MT Raiji', RC Langan², S Lanzara1, M Portinari', U Maestroni³, GM Palini', MV Zanzi', S

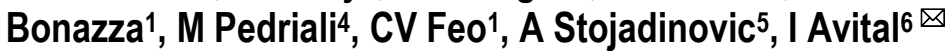

1. Section of General Surgery, Department of Surgical, Anaesthesiological and Radiological Sciences, Azienda Ospedaliero-Universitaria, Arcispedale Sant'Anna, Ferrara, Italy.

2. Department of Surgery, Georgetown University Hospital, Washington DC, USA.

3. Department of Surgery, University Hospital of Parma, Parma, Italy.

4. Section of Pathology, Department of Experimental and Diagnostic Medicine, University of Ferrara, Italy.

5. Department of Surgery, Division of Surgical Oncology, Walter Reed National Military Medical Center, Bethesda, MD, USA.

6. Bon Secours Cancer Institute, Bon Secours Hospital, Richmond VA, USA.

$\bowtie$ Corresponding author: Itzhak Avital, MD, FACS. Medical Director, Bon Secours Cancer Institute, Richmond VA 23223. itzhak_avital@bshsi.org.

( ) Ivyspring International Publisher. This is an open-access article distributed under the terms of the Creative Commons License (http://creativecommons.org/ licenses/by-nc-nd/3.0/). Reproduction is permitted for personal, noncommercial use, provided that the article is in whole, unmodified, and properly cited.

Received: 2012.06.13; Accepted: 2012.07.03; Published: 2012.07.25

\begin{abstract}
One in twelve American women will develop breast cancer, with infiltrating lobular carcinoma (ILC) comprising approximately I5\% of these cases. The incidence of ILC has been increasing over the last several decades. It has been hypothesized that this increase is associated with combined replacement hormonal therapy. Although pathologically distinct from infiltrating ductal carcinoma (IDC), ILC is treated in the same manner as IDC. However, ILC demonstrates significantly different patterns of late local recurrence and distant metastasis. The incidence of extra-hepatic gastrointestinal metastases is reported to be $6 \%$ to $18 \%$, with stomach being most common. Herein, we present a brief review of the literature and a typical case involving ILC initially presenting as a small bowel obstruction. Evidence suggests that the late clinical patterns of ILC are distinctly separate from IDC and physicians need be cognizant of its late local recurrence and unique late metastatic pattern. Different follow up strategy should be entertained in patients with ILC.
\end{abstract}

Key words: breast cancer, infiltrating lobular carcinoma

\section{Introduction}

Breast cancer holds the highest incidence of cancer in American women (30\% of all cancers) [1, 2]. Of these, 5 to $15 \%$ are comprised of infiltrating lobular carcinoma (ILC) [3]. ILC is more likely to be multicentric, bilateral and has an increasing incidence in postmenopausal women [4-7]. Although pathologically distinct, ILC appears to have similar clinical outcomes and prognosis as infiltrating ductal carcinoma (IDC) of the breast $[8,9]$. Studies of limited scope suggest that treatment for ILC should follow the guidelines for IDC [10]. However, others suggest that ILC is a different disease that should be treated and followed differently than IDC. ILC diverges significantly from IDC in terms of patterns of late local recurrence and sites of distant metastases [11, 12]. Metastatic IDC commonly involves the lung, bones and liver, whereas ILC more often involves the gastrointestinal (GI) tract, peritoneal surface, and retroperitoneum $[13,14]$. We present a brief review of the relevant literature discussing the association of ILC 
and late, gastrointestinal metastases often manifested as a gastrointestinal obstruction. To emphasize certain aspect of this disease, we present a 73-year old female with no oncologic history presenting with gastrointestinal obstruction, later found to be a pelvic mass and diffuse carcinomatosis due to ILC.

\section{Atypical presentation of a patient with late ILC gastrointestinal metastases:}

A 73-year old female presented with abdominal pain, nausea and emesis. Past medical and surgical history was significant for a right modified radical mastectomy thirty years prior to presentation. Pathology at that time was found to be benign. On current presentation, a computed-tomography (CT) scan was performed, demonstrating an 8 centimeter pelvic mass. Colonoscopy was performed and a stricture 15 centimeters above the anal verge was encountered. Multiple biopsies were taken, but were non-diagnostic. The patient's abdomen was then explored. Diffuse carcinomatosis was identified. Biopsies from the pelvic mass, omentum, and hepatic ligament were taken. The diagnosis of metastatic ILC was made through gross pathologic and immunohistochemistry (IHC) staining.

Subsequently, bilateral mammography and breast ultrasound was performed. A 5-millimeter lesion in the upper outer quadrant of the left breast was identified (the patient had a remote history of right breast abnormality that was resected and confirmed to be a benign disease). Core biopsy confirmed a primary ILC. IHC results were as follows: Strongly estrogen receptor positive $(97.5 \%)$, weakly progesterone receptor positive $(12.5 \%)$, Her-2/NEU negative $(0 \%)$, and MIB-1 labeling index of $0.5 \%$. The patient was then started on daily letrozole for 3 months and administered a single cycle of doxorubicin.

One year later, the patient developed symptoms of intestinal obstruction. Barium enema revealed a near complete obstruction at the site of the distal sigmoid stricture. An endoscopic stent was placed to relieve the obstruction. This was followed by left colectomy and diverting end colostomy. Pathologic examination of the surgical specimen demonstrated metastatic ILC with mild nuclear atypia and plasmacytoid features (Figure 1). Direct invasion of cells into the muscularis externa was present, with positive pan-cytokeratin staining (Figure 2). The patient continued to deteriorate postoperatively, and eventually succumbed to her disease.

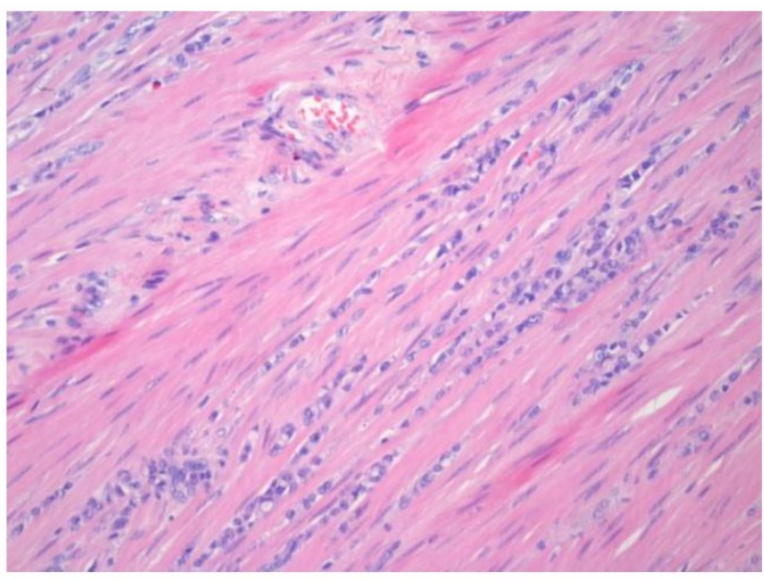

Figure I. Hematoxylin and eosin staining of the colonic specimen, demonstrating strands of invasive cells into the muscularis externa.

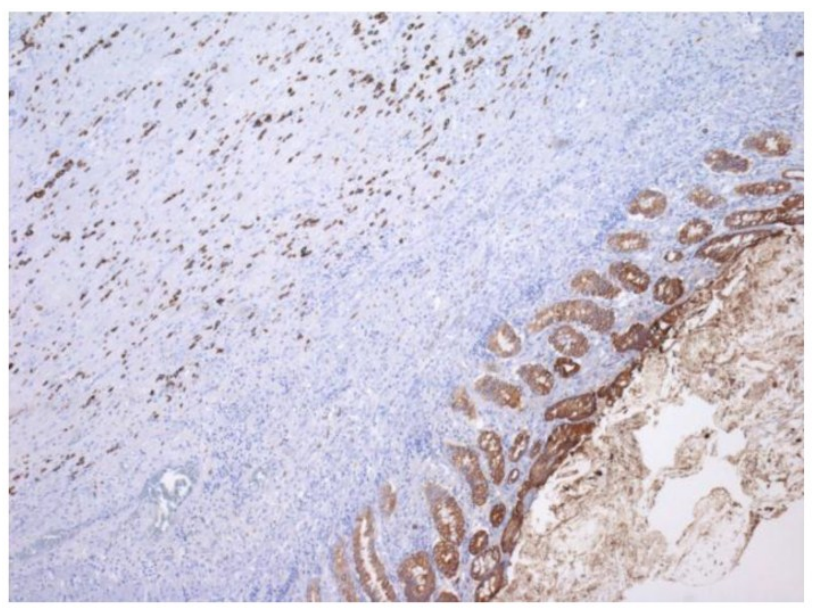

Figure 2. Immunohistochemical staining for cytokeratin, demonstrating individual invasive cells in the muscularis, with a similar staining intensity to the glandular epithelium of the colon.

\section{Review of the literature and discussion}

Breast cancer continues to be the most common carcinoma diagnosed in women, and is the second leading cause of cancer deaths in women [2]. ILC is the second most common histology of invasive breast cancers diagnosed $(15 \%)$, and has a rising incidence in postmenopausal women [7], which may be related to hormone replacement therapy [15]. ILC is more likely to be multicentric and bilateral [4-7]. Due to standardized screening protocols and improved imaging technology, only $10 \%$ of breast cancers present as metastatic disease $[16,17]$. In comparing the metastatic patterns of ILC versus IDC, Borst et al. found that ILC was significantly more likely to spread to the GI tract, gynecologic organs, peritoneal surface, and retroperitoneum [18]. Additionally, mammographic 
findings are often nonspecific [19, 20], leading to a known propensity for ILC to present at a later stage, with synchronous bilateral or metastatic disease [21]. Although pathologically distinct, ILC appears to have similar clinical outcomes and prognosis as infiltrating ductal carcinoma (IDC) of the breast $[8,9]$. This has led to the suggestion that ILC be treated in the same multimodality fashion as IDC [10]. However, due to the unique characteristics of ILC, we propose a closer follow up incorporating a strategy for GI tract evaluation to be implemented for patients with ILC.

Local failure in ILC has been reportedly decreased after total mastectomy as compared to breast conservation therapy [22], while others report that breast conservation does not impact local recurrence rates [23]. It is clear that ILC confers a greater risk of late local recurrence [24]. The rate of metastatic involvement of axillary lymph nodes in ILC appears to be similar to IDC [25-27]. This data suggests that primary operative management of ILC can be approached similarly to IDC [28].

Late development of metastatic disease in unique anatomical locations is a known characteristic of ILC. In particular, ILC metastases to the GI tract and peritoneum is a well-described entity [29]. Along with a primary intestinal malignancy, there are several cancers with a known propensity to metastasize to the gastrointestinal tract. Counted amongst this rare group of GI metastatic tumors are breast cancers, which represented $8 \%$ of secondary metastatic malignancies of the gastrointestinal tract in a surgical series [30]. Schwarz et al. reported on seven patients with a history of breast cancer who presented with GI metastasis ranging from 3 months to 12.5 years after initial diagnosis [31]. Of note, $4 / 7(57 \%)$ of these patients had originally been diagnosed with ILC. In a retrospective review of GI metastasis from breast cancer, $54 \%$ of metastatic breast cancers were of a lobular histology, far exceeding the $12 \%$ incidence of ILC diagnosed over the same time period [17]. Pestalozzi et al. showed that late recurrences caused a significant decline in disease free survival from ILC compared to IDC after approximately 6 years following curative treatment [32]. This tendency for late failures in ILC has been reported elsewhere [21, 33] and demonstrates that ILC tends to recur over 5 years after initial treatment.

Although rare, instances of occult ILC presenting initially with GI disease have been reported [34, 35]. Case reports of GI metastases as the initial presentation of ILC include lesions in the stomach, rectum, peritoneum, and/or retroperitoneum [16, 36, 37]. ILC presenting initially as a partially obstructing colonic mass with concomitant carcinomatosis has also been described [38]. In a retrospective review of 981 patients with ILC, Iorfida et al. demonstrated that tumor size greater than $2 \mathrm{~cm}$, positive axillary lymph nodes, and positive HER2 status were independent risk factors for metastatic disease [39]. Notably, our patient's primary tumor lacked any of these ominous indicators.

Given the propensity of ILC to develop late metastasis in the peritoneal cavity and retroperitoneum, we would recommend prolonged follow-up for patients with a known history of ILC using 3-dimensional axial imaging of the abdomen and pelvis. Further prospective evaluation of patients treated for ILC is necessary to determine the ideal interval and duration of follow-up. Additionally, it is important to consider metastatic ILC in the diagnostic evaluation of peritoneal surface malignancies (PSM). Although metastasis from an ovarian or intestinal primary [40] or primary peritoneal tumors [41] are high on the differential for PSM, our review of the literature demonstrates that a high index of suspicion for metastatic ILC must be maintained in this situation.

Peritoneal involvement from metastatic breast cancer carries a poor prognosis, with a median survival (MS) in a series of 44 patients reported by Tuthill et al. of 1.56 months, as compared to a 20.5 month MS for patients with metastatic breast cancer at other sites [42]. Due to the rarity of this disease entity, there is no standardized approach to PSM arising from a primary breast cancer. Controversy exists over the management of PSM arising from other primary sources, although current approaches to gynecologic [43] and intestinal $[44,45]$ PSM suggest that complete cytoreduction and heated intraperitoneal chemotherapy may provide a survival advantage. Evaluation of aggressive cytoreduction in patients with carcinomatosis of unknown primary suggests some benefit in terms of palliation and possibly survival [46]. While there are no studies evaluating aggressive surgical management of PSM arising from ILC, this case report raises an interesting prospective area of study.

\section{Conclusion}

ILC of the breast is a distinct pathologic entity as compared to IDC. ILC harbors increased potential for late local recurrence and late metastatic disease to gastrointestinal track. Unlike IDC, which typically metastasized to the lung, liver or bone, ILC has been found to metastasize to the GI tract, peritoneum and retroperitoneum $[13,14]$. To illustrate these situations, we presented a typical case of ILC metastatic spread to the GI tract and peritoneal surface. We propose that in patients with a known history of ILC, a high index 
of suspicion for metastatic disease, in spite of a prolonged disease-free interval, needs to be entertained. In addition, metastatic ILC should remain high on the differential in female patients presenting with GI tract malignancies of unknown origin. Physicians need to be cognizant of this metastatic masquerade and we advocate closer follow up and specifically more intense GI follow up for patients diagnosed with ILC.

\section{Contributing Author Declaration}

We certify that all individuals who qualify as authors have been listed; each has participated in one or more of the following areas: conception and design of this work, the acquisition and/or analysis of data, the writing, and/or critical revision of the document, and supervision of this cooperative research effort. All contributing authors approve of the submission of this version of the manuscript and assert that the document represents valid work. If information derived from another source was used in this manuscript, we obtained all necessary approvals to use it and made appropriate acknowledgements in the document. All contributing authors take public responsibility for this work.

\section{Competing Interests}

The authors have declared that no competing interest exists.

\section{References}

1. Siegel R, Ward E, Brawley O, et al. Cancer statistics, 2011: the impact of eliminating socioeconomic and racial disparities on premature cancer deaths. CA Cancer J Clin. 2011; 61(4): 212-36.

2. Siegel R, Naishadham D, and Jemal A. Cancer statistics, 2012. CA Cancer J Clin. 2012; 62(1): 10-29.

3. Li CI, Uribe DJ, and Daling JR. Clinical characteristics of different histologic types of breast cancer. Br J Cancer. 2005; 93(9): 1046-52.

4. Fortunato L, Mascaro A, Poccia I, et al. Lobular breast cancer: same survival and local control compared with ductal cancer, but should both be treated the same way? analysis of an institutional database over a 10-year period. Ann Surg Oncol. 2012; 19(4): 1107-14.

5. Intra $\mathrm{M}$, Rotmensz $\mathrm{N}$, Viale $\mathrm{G}$, et al. Clinicopathologic characteristics of 143 patients with synchronous bilateral invasive breast carcinomas treated in a single institution. Cancer. 2004; 101(5): 905-12.

6. Lesser ML, Rosen PP, and Kinne DW. Multicentricity and bilaterality in invasive breast carcinoma. Surgery. 1982; 91(2): 234-40.

7. $\mathrm{Li} \mathrm{CI}$, Anderson $\mathrm{BO}$, Porter $\mathrm{P}$, et al. Changing incidence rate of invasive lobular breast carcinoma among older women. Cancer. 2000; 88(11): 2561-9.

8. Kurtz JM, Jacquemier J, Torhorst J, et al. Conservation therapy for breast cancers other than infiltrating ductal carcinoma. Cancer. 1989; 63(8): 1630-5.

9. Winchester DJ, Chang HR, Graves TA, et al. A comparative analysis of lobular and ductal carcinoma of the breast: presentation, treatment, and outcomes. J Am Coll Surg. 1998; 186(4): 416-22.

10. Santiago RJ, Harris EE, Qin L, et al. Similar long-term results of breast-conservation treatment for Stage I and II invasive lobular carcinoma compared with invasive ductal carcinoma of the breast: The University of Pennsylvania experience. Cancer. 2005; 103(12): 2447-54.
11. Anwar IF, Down SK, Rizvi S, et al. Invasive lobular carcinoma of the breast: should this be regarded as a chronic disease? Int J Surg. 2010; 8(5): 346-52.

12. Harris M, Howell A, Chrissohou M, et al. A comparison of the metastatic pattern of infiltrating lobular carcinoma and infiltrating duct carcinoma of the breast. Br J Cancer. 1984; 50(1): 23-30.

13. Ferlicot S, Vincent-Salomon A, Medioni J, et al. Wide metastatic spreading in infiltrating lobular carcinoma of the breast. Eur J Cancer. 2004; 40(3): 336-41.

14. Winston $\mathrm{CB}$, Hadar $\mathrm{O}$, Teitcher $\mathrm{JB}$, et al. Metastatic lobular carcinoma of the breast: patterns of spread in the chest, abdomen, and pelvis on CT. AJR Am J Roentgenol. 2000; 175(3): 795-800.

15. Eheman $C R$, Shaw $K M$, Ryerson $A B$, et al. The changing incidence of in situ and invasive ductal and lobular breast carcinomas: United States, 1999-2004. Cancer Epidemiol Biomarkers Prev. 2009; 18(6): 1763-9.

16. Neal L, Sookhan N, and Reynolds C. Occult breast carcinoma presenting as gastrointestinal metastases. Case Report Med. 2009; 2009: 564756.

17. McLemore EC, Pockaj BA, Reynolds C, et al. Breast cancer: presentation and intervention in women with gastrointestinal metastasis and carcinomatosis. Ann Surg Oncol. 2005; 12(11): 886-94.

18. Borst MJ and Ingold JA. Metastatic patterns of invasive lobular versus invasive ductal carcinoma of the breast. Surgery. 1993; 114(4): 637-41.

19. Le Gal M, Ollivier L, Asselain B, et al. Mammographic features of 455 invasive lobular carcinomas. Radiology. 1992; 185(3): 705-8.

20. Krecke KN and Gisvold JJ. Invasive lobular carcinoma of the breast: mammographic findings and extent of disease at diagnosis in 184 patients. AJR Am J Roentgenol. 1993; 161(5): 957-60.

21. Arpino G, Bardou VJ, Clark GM, et al. Infiltrating lobular carcinoma of the breast: tumor characteristics and clinical outcome. Breast Cancer Res. 2004; 6(3): R149-56.

22. Hussien M, Lioe TF, Finnegan J, et al. Surgical treatment for invasive lobular carcinoma of the breast. Breast. 2003; 12(1): 23-35.

23. Stolier AJ, Barre G, Bolton JS, et al. Breast conservation therapy for invasive lobular carcinoma: the impact of lobular carcinoma in situ in the surgical specimen on local recurrence and axillary node status. Am Surg. 2004; 70(9): 818-21.

24. Cocquyt V and Van Belle S. Lobular carcinoma in situ and invasive lobular cancer of the breast. Curr Opin Obstet Gynecol. 2005; 17(1): 55-60.

25. Gann PH, Colilla SA, Gapstur SM, et al. Factors associated with axillary lymph node metastasis from breast carcinoma: descriptive and predictive analyses. Cancer. 1999; 86(8): 1511-9.

26. Voogd AC, Coebergh JW, Repelaer van Driel OJ, et al. The risk of nodal metastases in breast cancer patients with clinically negative lymph nodes: a population-based analysis. Breast Cancer Res Treat. 2000; 62(1): 63-9.

27. Vandorpe T, Smeets A, Van Calster B, et al. Lobular and non-lobular breast cancers differ regarding axillary lymph node metastasis: a cross-sectional study on 4,292 consecutive patients. Breast Cancer Res Treat. 2011; 128(2): 429-35.

28. Sastre-Garau X, Jouve M, Asselain B, et al. Infiltrating lobular carcinoma of the breast. Clinicopathologic analysis of 975 cases with reference to data on conservative therapy and metastatic patterns. Cancer. 1996; 77(1): 113-20.

29. Dixon AR, Ellis IO, Elston CW, et al. A comparison of the clinical metastatic patterns of invasive lobular and ductal carcinomas of the breast. Br J Cancer. 1991; 63(4): 634-5.

30. Washington $\mathrm{K}$ and McDonagh $\mathrm{D}$. Secondary tumors of the gastrointestinal tract: surgical pathologic findings and comparison with autopsy survey. Mod Pathol. 1995; 8(4): 427-33.

31. Schwarz RE, Klimstra DS, and Turnbull AD. Metastatic breast cancer masquerading as gastrointestinal primary. Am J Gastroenterol. 1998; 93(1): 111-4.

32. Pestalozzi BC, Zahrieh D, Mallon E, et al. Distinct clinical and prognostic features of infiltrating lobular carcinoma of the breast: combined results of 15 International Breast Cancer Study Group clinical trials. J Clin Oncol. 2008; 26(18): 3006-14. 
33. Lee JH, Park S, Park HS, et al. Clinicopathological features of infiltrating lobular carcinomas comparing with infiltrating ductal carcinomas: a case control study. World J Surg Oncol. 2010; 8: 34.

34. Mistrangelo M, Cassoni P, Castellano I, et al. Obstructive colon metastases from lobular breast cancer: report of a case and review of the literature. Tumori. 2011; 97(6): 800-4.

35. Nazareno J, Taves D, and Preiksaitis HG. Metastatic breast cancer to the gastrointestinal tract: a case series and review of the literature. World J Gastroenterol. 2006; 12(38): 6219-24.

36. Arrangoiz R, Papavasiliou P, Dushkin $\mathrm{H}$, et al. Case report and literature review: Metastatic lobular carcinoma of the breast an unusual presentation. Int J Surg Case Rep. 2011; 2(8): 301-5.

37. Clavien PA, Laffer U, Torhost J, et al. Gastro-intestinal metastases as first clinical manifestation of the dissemination of a breast cancer. Eur J Surg Oncol. 1990; 16(2): 121-6.

38. Saranovic D, Kovac JD, Knezevic S, et al. Invasive lobular breast cancer presenting an unusual metastatic pattern in the form of peritoneal and rectal metastases: a case report. J Breast Cancer. 2011; 14(3): 247-50.

39. Iorfida $\mathrm{M}$, Maiorano $\mathrm{E}$, Orvieto $\mathrm{E}$, et al. Invasive lobular breast cancer: subtypes and outcome. Breast Cancer Res Treat. 2012 Jun;133(2):713-23.

40. Levy AD, Shaw JC, and Sobin LH. Secondary tumors and tumorlike lesions of the peritoneal cavity: imaging features with pathologic correlation. Radiographics. 2009; 29(2): 347-73.

41. Levy AD, Arnaiz J, Shaw JC, et al. From the archives of the AFIP: primary peritoneal tumors: imaging features with pathologic correlation. Radiographics. 2008; 28(2): 583-607.

42. Tuthill M, Pell R, Guiliani R, et al. Peritoneal disease in breast cancer: a specific entity with an extremely poor prognosis. Eur J Cancer. 2009; 45(12): 2146-9.

43. Bijelic L, Jonson A, and Sugarbaker PH. Systematic review of cytoreductive surgery and heated intraoperative intraperitoneal chemotherapy for treatment of peritoneal carcinomatosis in primary and recurrent ovarian cancer. Ann Oncol. 2007; 18(12): 1943-50.

44. Cole KL, Choudry HA, Jones HL, et al. Critical role of hyperthermic intraperitoneal chemoperfusion in the treatment of a patient with Pseudomyxoma peritonei. J Surg Oncol. 2012; doi: $10.1002 /$ jso.23088

45. Verwaal VJ, Bruin S, Boot H, et al. 8-year follow-up of randomized trial: cytoreduction and hyperthermic intraperitoneal chemotherapy versus systemic chemotherapy in patients with peritoneal carcinomatosis of colorectal cancer. Ann Surg Oncol. 2008; 15(9): 2426-32.

46. Sebbag G, Shmookler BM, Chang D, et al. Peritoneal carcinomatosis from an unknown primary site. Management of 15 patients. Tumori. 2001; 87(2): 67-73. 